\title{
Study on Stability for Yield and Its Components in Tomato (Solanum lycopersicum L.)
}

\author{
A. Shankar*, R.V.S.K. Reddy, P. Saidaiah, K. Uma Krishna and K. Uma Jyothi \\ Horticultural college and research institute, V. R. Gudem, Dr.Y.S.R.HU, \\ West Godavari, A.P., India \\ *Corresponding author
}

Keywords

Stability, Yield,

Component,

Tomato.

Article Info

Accepted:

14 July 2017

Available Online:

10 September 2017

\section{A B S T R A C T}

The present investigation "Study on stability for yield and its components in tomato (Solanum lycopersicum L.)" was carried out in rabi, kharif and summer at Vegetable Research Station, Rajendranagar, Hyderabad. Six parents LE-53, LE62, LE-64, Arka Alok, Arka Meghali and Arka Vikas were crossed in diallel mating design. The resultant $30 \mathrm{~F}_{1}$ 's were evaluated along with their parents and two standard checks (Lakshmi and US-618) for characters viz., plant height $(\mathrm{cm})$, number of primary branches per plant, days to $50 \%$ flowering, number of flowers per cluster, number of fruits per cluster, fruit set (\%), days to first fruit harvest, days to last fruit harvest, fruit yield per plant $(\mathrm{kg})$. Results of stability analysis revealed that the hybrids viz., Arka Vikas $\times$ Arka Meghali, Arka Meghali $\times$ LE-64, Arka Vikas $\times$ Arka Alok, LE-62 $\times$ LE-53, LE-64 × Arka Meghali, Arka Meghali $\times$ Arka Alok and LE-62 $\times$ Arka Meghali were identified as stable and best performing hybrids for fruit yield per plant and other traits hence, suitable for wider environments. Among the parents, LE-62 was the stable with high per se performance for yield per plant.

\section{Introduction}

Tomato (Solanum lycopersicum L., $2 \mathrm{n}=2 \mathrm{x}=$ 24, Family: Solanaceae) is usually a fruit vegetable. Throughout the world, after potato, it is a popular and widely grown vegetable (FAOSTAT, 2015) because of its wider adaptability, high yielding potential and suitability for variety of uses in fresh as well as processed food industries.

In India, tomato is grown in an area of 0.882 million hectares with annual production of 18.74 million tonnes and productivity of 21.2 tonnes/ha (NHB, 2015).
The scenario of tomato production in the country has tremendously changed over the past few decades with increasing popularity of hybrids. For commercialization of new hybrids, it requires stable genotypes. Genotype $\times$ environment interactions are known to interferes with evaluation of genotypes and reduce the progress of selection in a plant breeding programme.

Hence, estimation of the nature and magnitude of genotype $\times$ environment interactions for yield and yield components is 
essential to identify a stable genotype over environments.

In view of the above, the present investigation was conducted by crossing six elite parents in full diallel mating design. The resulted 30 $\mathrm{F}_{1}$ 's, parents and two commercial checks (Lakshmi and US-618) were evaluated in RBD design over three seasons (kharif, rabi and summer) at VRS, Rajendranagar, Dr. YSRHU to identify stable hybrids

\section{Materials and Methods}

The present investigation was conducted by crossing six elite parents (LE-53, LE-62, LE64, Arka Alok, Arka Meghali and Arka Vikas) in full diallel mating design. The resulted $30 \mathrm{~F}_{1}$ 's, parents and two commercial checks (Lakshmi and US-618) were evaluated in RBD design over three seasons (kharif, rabi and summer) at VRS, Rajendranagar, Dr. YSRHU to identify stable hybrids. The data was recorded for characters viz., plant height $(\mathrm{cm})$, number of primary branches per plant, days to $50 \%$ flowering, number of flowers per cluster, number of fruits per cluster, fruit set (\%), days to first fruit harvest, days to last fruit harvest, fruit yield per plant $(\mathrm{kg})$. Data obtained from the three seasons were subjected to pooled analysis of variance (Panse and Sukhatme, 1985). The genotype $x$ environment interactions was significant for most of the characters and hence the data was further analyzed to determine the phenotypic stability of different hybrids.

Eberhart and Russell (1966) methodology used to study the stability of genotypes under different environments. In this methodology three parameters namely (i) overall mean of each genotype over a range of environments $(\mu \mathrm{i})$, (ii) the regression of each genotype on the environmental index (bi) and (iii) a function of the squared deviation from the regression $\left(\mathrm{S}^{2} \mathrm{~d}_{\mathrm{i}}\right)$ were estimated.
Yij $\quad=\mu i+b i \mathrm{Ij}+\delta i j$

\section{Results and Discussion}

According to Eberhart and Russell (1966), the ideal genotype would be the one which has high mean value, unit regression coefficient $\left(b_{i}=1\right)$ and minimum deviation from regression $\left(S^{2} d_{i}=0\right)$. The linear regression $\left(b_{i}\right)$ is treated as a measure of response of a genotype and deviation from regression $\left(\mathrm{S}^{2} \mathrm{~d}_{\mathrm{i}}\right)$ is considered as a measure of stability. In the present study, if regression coefficient $\left(b_{i}\right)$ values, found non-significant are treated as unity. If deviation from regression $\left(\mathrm{S}^{2} \mathrm{~d}_{\mathrm{i}}\right)$ values, found non-significant are considered to be within the "minimum deviation" i.e., zero, whose genotypes were statistically within the range of minimum deviation from regression and performance can be predicted. Hence, the genotypes which possess the above values are considered to be stable.

\section{Pooled analysis of variance for stability}

The ANOVA recorded significant differences among genotypes (Table 1). Mean sum of squares due to environments was significant for all the traits except plant height which indicated the strong influence of environment on genotypes. The genotypes were significantly differed for days to $50 \%$ flowering and no. of flowers per cluster which showed the wide variability for these traits over seasons. None of the traits recorded significant mean sum of squares due to genotypic $\mathrm{x}$ environment interactions. Partitioning of mean squares due to environments + (genotypes $\mathrm{x}$ environments) were significant for all the characters emphasizing the existence of $\mathrm{G} \times \mathrm{E}$ interactions for all the traits.

Sum of squares due to $E+(G \times E)$ was further partitioned into that of environment (linear), genotype $\times$ environment (linear) and pooled 
deviation. Significant variation due to environment (linear) and genotype $\times$ environment (linear) was observed for all the traits except plant height indicated the linear contribution of environmental effects and additive environment variance on these characters and the genotypes significantly differing for their linear response to environments, respectively. The mean sum of squares for pooled deviation was significant for all traits indicating the non-linear response and unpredictable nature of genotypes by significantly differing for stability.

\section{Plant height (cm)}

Among parents, Arka Meghali recorded regression coefficient $\left(b_{i}\right)$ and deviation from regression $\left(S^{2} d_{i}\right)$ values were non-significant hence considered to be widely adaptable to different environments with average stability.

Among the eighteen stable hybrids, none could record significantly higher plant height than the best check US-618 $(80.93 \mathrm{~cm})$. However, Arka Meghali × LE-64 $(74.15 \mathrm{~cm})$ and LE-62 $\times$ LE-53 $(72.36 \mathrm{~cm})$ were at par for plant height with the best check US-618. The hybrid LE-62 $\times$ LE-64 $(69.05 \mathrm{~cm})$ recorded $b_{i}$ value greater than unity and thus considered to possess less than the average stability and it is adaptable to favourable environments. Similar results were reported by Kalloo et al., (1998), Mulge and Aravindakumar (2003) and Mandal et al., (2000) in tomato.

\section{Number of primary branches per plant}

Among parents, LE-53, LE-64 and Arka Meghali exhibited unit $b_{i}$ value with $S^{2} d_{i}$ equal to zero found to have average stability and widely adaptable for this trait. The parent, Arka Alok recorded regression coefficient $\left(b_{i}\right)$ more than unit with minimum deviation thus considered to possess less than the average stability and adaptability to favourable environments.

Among the stable hybrids, the hybrid Arka Vikas $\times$ Arka Alok (5.21) recorded significantly higher no. of primary branches per plant than best check Lakshmi (4.54). However eight hybrids exhibited at par for no. of primary branches per plant with the best check, Lakshmi and average stability suggesting wider adaptability and their performance does not change with change in environments. Similar findings were also reported by Pandey (1983), Mulge and Aravindakumar (2003) and Prasanna et al., (2007), who reported significant genotype $x$ environment linear interaction for no. of primary branches per plant (Table 2).

\section{Days to 50\% flowering}

Among the parents, LE-64 (28 days) showed significantly early flowering than best check Lakshmi (29.89 days) with less than unit regression coefficient $\left(b_{i}<1\right)$ indicating more than the average stability and adaptability to poor environments.

Among the fourteen hybrids, LE-53 $\times$ LE-64 (24.33 days), Arka Vikas $\times$ Arka Meghali (24.56 days), Arka Vikas $\times$ LE-62 (24.78 days), Arka Meghali $\times$ LE-53 (25.22 days), Arka Meghali $\times$ Arka Vikas (26.67 days) and LE-62 $\times$ LE-53 (26.78 days) recorded significantly less number of days to $50 \%$ flowering than the best check, Lakshmi (29.89 days) with unit regression values. Hence, they were considered to be stable hybrids and recommended for wider environments. The hybrid, LE-64 $\times$ LE-62 (26.33 days) and check Lakshmi (29.89 days) recorded significantly less no. of days to $50 \%$ flowering coupled with greater than unity regression coefficient value. Hence it can be recommended as stable hybrid for favorable environment. 
Table.1 ANOVA of stability for yield and yield contributing traits in tomato

\begin{tabular}{|c|c|c|c|c|c|c|c|c|c|c|}
\hline \multirow{3}{*}{$\begin{array}{l}\text { S. } \\
\text { No. }\end{array}$} & \multirow{3}{*}{ Characters } & & \multicolumn{8}{|c|}{ Mean sum of squares } \\
\hline & & & $\begin{array}{l}\text { Rep } \\
\text { within } \\
\text { Env. }\end{array}$ & Genotypes & $\begin{array}{l}\text { Env. }+ \\
\text { (Genotype } \\
\times \text { Env.) }\end{array}$ & Environments & $\begin{array}{l}\text { Genotypes } \\
\times \text { Env. }\end{array}$ & $\begin{array}{l}\text { Environments } \\
\text { (Lin.) }\end{array}$ & $\begin{array}{c}\text { Genotypes } \\
\times \\
\text { Env.(Lin.) }\end{array}$ & $\begin{array}{l}\text { Pooled } \\
\text { Deviation }\end{array}$ \\
\hline & & df & 6 & 37 & 76 & 2 & 74 & 1 & 37 & 38 \\
\hline 1 & Plant height $(\mathrm{cm})$ & & 14.73 & 129 & 100.87 & 16.59 & 103.15 & 33.17 & 50.81 & $151.40^{* *}$ \\
\hline 2 & $\begin{array}{l}\text { No. of primary branches/ } \\
\text { plant }\end{array}$ & & 0.03 & 0.28 & $0.72 *$ & $11.17 * *$ & 0.44 & $21.85 * *$ & $0.95 * *$ & $0.42 * *$ \\
\hline 3 & Days to $50 \%$ flowering & & 0.62 & $16.93 * *$ & $10.42 *$ & $130.84 * *$ & 7.17 & $256.17 * *$ & $13.70 *$ & $5.97 * *$ \\
\hline 4 & No. of flowers/ cluster & & 0.04 & $0.19 *$ & $0.64 * *$ & $19.00 * *$ & 0.15 & $38.01 * *$ & $0.19 *$ & $0.10 * *$ \\
\hline 5 & No. of fruits/ cluster & & 0.03 & 0.17 & $0.92 * *$ & $31.14 * *$ & 0.1 & $61.46^{* *}$ & $0.91 *$ & $0.11 * *$ \\
\hline 6 & Fruit set $(\%)$ & & 2.72 & 61.1 & $333.61 * *$ & $10869.83 * *$ & 48.85 & $21696.70 * *$ & $95.62 * *$ & $43.87 * *$ \\
\hline 7 & Days to first harvest & & 0.04 & 0.14 & $0.24 * *$ & $5.45 * *$ & 0.1 & $10.77 * *$ & $0.25 * *$ & $0.08 * *$ \\
\hline 8 & Days to last harvest & & 0.9 & 121 & $189.90 * *$ & $5541.80 * *$ & 45.26 & $11013.34 * *$ & $83.53 * *$ & $75.20 * *$ \\
\hline 9 & Fruit yield/ plant (kg) & & 0.01 & 5.91 & 3.19 & $25.17 *$ & 2.6 & $43.93 * *$ & $6.52 * *$ & $4.94 * *$ \\
\hline
\end{tabular}


Table.2 Mean performance and stability parameters for yield and its components in tomato

\begin{tabular}{|c|c|c|c|c|c|c|c|c|c|c|c|c|c|c|c|}
\hline \multirow[t]{2}{*}{ Parents $(\mathbf{P}) /$ Crosses $(\mathrm{C})$} & \multicolumn{3}{|c|}{ Plant height $(\mathbf{c m})$} & \multicolumn{3}{|c|}{ No. of primary branches/plant } & \multicolumn{3}{|c|}{ Days to $50 \%$ flowering } & \multicolumn{3}{|c|}{ No. of flowers per cluster } & \multicolumn{3}{|c|}{ No. of fruits/ cluster } \\
\hline & $\mu$ Mean & $\mathbf{b}_{\mathbf{i}}$ & $\mathbf{S}^{2} \mathbf{d}_{\mathrm{i}}$ & $\mu$ Mean & $\mathbf{b}_{\mathbf{i}}$ & $\mathbf{S}^{2} \mathbf{d}_{\mathrm{i}}$ & $\mu$ Mean & $\mathbf{b}_{\mathbf{i}}$ & $\mathbf{S}^{2} \mathbf{d}_{\mathrm{i}}$ & $\mu$ Mean & $\mathbf{b}_{\mathbf{i}}$ & $\mathbf{S}^{2} \mathbf{d}_{\mathrm{i}}$ & $\mu$ Mean & $\mathbf{b}_{\mathbf{i}}$ & $\mathbf{S}^{2} \mathbf{d}_{\mathrm{i}}$ \\
\hline LE-53 (P1) & 61.72 & 4.64 & $146.38 * *$ & 4.72 & -0.72 & 0.03 & 26.00 & 1.91 & $12.45 * *$ & 4.13 & 0.89 & 0.04 & 2.03 & 0.95 & -0.02 \\
\hline LE-62 (P2) & 72.47 & -5.67 & $268.93 * *$ & 4.99 & 0.68 & $0.30^{*}$ & 29.67 & -0.91 & $2.12 *$ & 3.81 & 0.39 & $0.09 *$ & 2.04 & 0.83 & 0.07 \\
\hline LE-64 (P3) & 64.69 & -12.37 & $69.33 *$ & 4.46 & 1.16 & 0.13 & 28.00 & $0.00 * *$ & -0.41 & 4.02 & 1.06 & 0.01 & 2.17 & 0.72 & -0.02 \\
\hline Arka Alok (P4) & 57.05 & -2.72 & -4.87 & 4.09 & $1.21 * *$ & -0.06 & 27.78 & 1.74 & $29.83 * *$ & 3.73 & 1.45 & -0.01 & 2.26 & 1.29 & -0.02 \\
\hline Arka Meghali (P5) & 70.53 & -13.23 & $629.24 * *$ & 4.44 & 0.48 & 0.17 & 32.78 & 0.97 & $11.48^{* *}$ & 4.09 & 0.66 & 0.06 & 2.34 & 1.23 & $0.09 *$ \\
\hline Arka Vikas (P6) & 71.65 & -15.23 & $192.03 * *$ & 4.29 & 1.71 & $1.55^{* *}$ & 32.00 & 0.25 & $13.18^{* *}$ & 3.54 & 0.92 & -0.01 & 2.04 & 1.17 & 0.03 \\
\hline LE-53 × LE-62 (C1) & 72.17 & 3.98 & $133.96^{* *}$ & 5.08 & 1.32 & -0.05 & 30.44 & 1.87 & $3.04 * *$ & 4.30 & 1.59 & $0.30 * *$ & 1.95 & 0.70 & -0.01 \\
\hline LE-53 × LE-64 (C2) & 81.85 & 9.51 & $249.71 * *$ & 4.58 & 1.32 & -0.06 & 24.33 & 2.16 & 0.10 & 3.96 & 0.87 & 0.07 & 2.06 & 0.62 & 0.07 \\
\hline LE-53 × A. Alok (C3) & 61.93 & -1.36 & $83.49 * *$ & 3.90 & 0.74 & $2.26^{* *}$ & 30.89 & 0.71 & 0.85 & 4.22 & 0.77 & $0.74 * *$ & 2.53 & 0.63 & $1.00^{* *}$ \\
\hline LE-53 × A. Meghali (C4) & 67.02 & 5.11 & $166.97 * *$ & 4.74 & 0.73 & $0.28 *$ & 31.00 & 0.71 & $2.37 * *$ & 3.76 & 1.22 & 0.03 & 1.97 & $0.79 * *$ & -0.02 \\
\hline LE-53 × A. Vikas (C5) & 66.65 & 1.00 & $49.31 *$ & 4.59 & -0.18 & $3.50 * *$ & 28.22 & 0.28 & $2.68 * *$ & 3.94 & 0.87 & -0.02 & 2.13 & 0.94 & 0.06 \\
\hline LE-62 × LE-53 (C6) & 72.36 & -6.48 & 2.37 & 4.82 & -1.12 & $0.22 *$ & 26.78 & 1.44 & -0.37 & 4.04 & 1.11 & $0.16^{*}$ & 2.14 & 1.13 & 0.06 \\
\hline LE-62 × LE-64 (C7) & 69.05 & $10.40^{*}$ & -11.92 & 4.32 & -0.55 & -0.04 & 24.44 & 1.55 & $8.95 * *$ & 3.85 & 0.60 & -0.02 & 2.25 & 1.01 & -0.02 \\
\hline LE-62 × A. Alok (C8) & 94.76 & 6.72 & $2140.06^{* *}$ & 4.79 & 0.81 & $0.79 * *$ & 22.67 & 1.64 & $15.64 * *$ & 4.35 & 1.18 & 0.00 & 2.60 & 1.49 & $0.36 * *$ \\
\hline LE-62 × A. Meghali (C9) & 62.43 & 5.10 & -8.79 & 4.71 & 0.49 & -0.05 & 28.67 & -0.01 & 0.26 & 3.72 & 0.15 & -0.01 & 2.11 & 0.55 & $0.09 *$ \\
\hline LE-62 × A. Vikas (C10) & 70.82 & -1.27 & $321.57 * *$ & 4.37 & 0.14 & $0.89 * *$ & 26.89 & 0.55 & $4.22 * *$ & 3.85 & 0.96 & -0.03 & 2.10 & 1.03 & $0.10^{*}$ \\
\hline LE-64 × LE-53 (C11) & 70.02 & -8.48 & -5.13 & 5.00 & 1.64 & $0.24 *$ & 28.33 & 1.08 & 0.18 & 4.22 & 2.01 & 0.05 & 2.12 & 0.92 & $0.17 * *$ \\
\hline LE-64 × LE-62 (C12) & 69.00 & -1.93 & $77.14 * *$ & 4.03 & 0.77 & -0.01 & 26.33 & $3.41^{*}$ & -0.38 & 4.05 & 0.27 & 0.00 & 2.19 & $1.18^{*}$ & -0.02 \\
\hline LE-64 × A. Alok (C13) & 68.48 & -2.63 & -8.78 & 4.54 & 1.23 & 0.00 & 27.56 & 1.23 & $12.52 * *$ & 4.23 & 0.64 & $0.26^{* *}$ & 2.30 & 1.09 & -0.02 \\
\hline LE-64 × A. Meghali (C14) & 68.80 & 2.28 & $221.98 * *$ & 4.31 & 1.56 & $0.22 *$ & 27.11 & 0.53 & $1.51^{*}$ & 4.06 & 0.97 & 0.08 & 2.42 & 1.11 & -0.01 \\
\hline LE-64 × A.Vikas (C15) & 67.14 & 9.06 & $184.55 * *$ & 4.15 & 0.26 & -0.06 & 28.11 & 2.01 & $17.76^{* *}$ & 4.09 & 0.90 & -0.01 & 2.16 & 0.99 & $0.14 * *$ \\
\hline A. Alok $\times$ LE-53 (C16) & 66.29 & -8.06 & -10.59 & 4.56 & 2.64 & $0.27 *$ & 31.78 & 1.90 & $1.58^{*}$ & 4.10 & $1.61 *$ & -0.03 & 2.61 & 0.80 & $0.29 * *$ \\
\hline A. Alok $\times$ LE-62 (C17) & 66.93 & -1.18 & -5.25 & 4.39 & 1.57 & 0.00 & 28.56 & 0.36 & -0.32 & 3.95 & 0.68 & $0.12 *$ & 2.38 & 1.02 & 0.06 \\
\hline A. Alok $\times$ LE-64 (C18) & 66.23 & -2.62 & -11.57 & 4.71 & 2.34 & 0.11 & 30.00 & 1.97 & 0.42 & 4.56 & 1.47 & 0.04 & 2.27 & $1.36^{*}$ & -0.02 \\
\hline A. Alok $\times$ A. Meghali (C19) & 63.00 & 4.51 & 13.01 & 4.58 & 2.01 & -0.04 & 25.89 & 1.45 & $1.27 *$ & 3.97 & 1.27 & 0.06 & 2.49 & 1.43 & $0.10^{*}$ \\
\hline A. Alok × A. Vikas (C20) & 70.30 & 1.94 & 7.61 & 4.85 & 1.24 & -0.01 & 29.56 & 0.11 & $6.25 * *$ & 4.24 & 2.23 & -0.01 & 2.80 & 0.86 & $0.61 * *$ \\
\hline A. Meghali × LE-53 (21) & 70.53 & -5.80 & $90.86^{* * *}$ & 4.37 & 2.64 & $1.88 * *$ & 25.22 & 2.33 & -0.26 & 3.60 & 1.36 & 0.00 & 2.11 & 1.08 & -0.01 \\
\hline A. Meghali $\times$ LE-62 (C22) & 68.26 & 4.38 & $39.47 *$ & 4.80 & 0.36 & $0.89^{* *}$ & 28.33 & 1.34 & -0.19 & 4.31 & 0.44 & $0.55^{* * *}$ & 2.04 & 1.17 & -0.02 \\
\hline A. Meghali $\times$ LE-64 (C23) & 74.15 & 4.53 & -12.18 & 4.54 & 0.75 & -0.06 & 28.56 & -1.72 & $1.60^{*}$ & 4.17 & 1.21 & 0.03 & 2.79 & 0.84 & $0.18^{* * *}$ \\
\hline A. Meghali × A. Alok (C24) & 67.57 & 5.06 & 0.96 & 4.88 & 0.98 & 0.09 & 28.33 & 0.10 & 1.08 & 3.67 & 0.87 & -0.03 & 2.26 & 1.35 & -0.01 \\
\hline A. Meghali $\times$ A. Vikas $(\mathrm{C} 25)$ & 66.98 & 11.63 & 13.50 & 4.76 & 1.11 & -0.06 & 26.67 & 3.23 & 0.53 & 3.46 & 0.80 & -0.01 & 2.05 & 0.89 & -0.02 \\
\hline A. Vikas $\times$ LE-53 (C26) & 69.75 & 6.53 & 17.32 & 4.47 & 1.82 & -0.06 & 30.22 & -0.13 & $40.43^{* *}$ & 3.86 & 0.98 & -0.01 & 2.05 & $1.05 * *$ & -0.02 \\
\hline A. Vikas × LE-62 (27) & 63.66 & 7.20 & -2.60 & 4.15 & 0.82 & -0.05 & 24.78 & 0.36 & -0.34 & 3.80 & 0.92 & -0.03 & 2.12 & 1.17 & -0.02 \\
\hline A. Vikas $\times$ LE-64 (C28) & 70.21 & 18.95 & 9.77 & 4.94 & 1.30 & -0.04 & 28.56 & -0.45 & 0.03 & 3.86 & 0.92 & 0.01 & 2.10 & 1.03 & -0.02 \\
\hline A. Vikas × A. Alok (C29) & 69.62 & -11.40 & $132.43 * *$ & 5.21 & 1.95 & -0.03 & 29.44 & 0.01 & $3.22 * *$ & 4.15 & 1.28 & -0.01 & 2.81 & 0.95 & $0.17 * *$ \\
\hline A. Vikas $\times$ A. Meghali $(\mathrm{C} 30)$ & 59.91 & 1.74 & 27.72 & 4.87 & -0.34 & 0.04 & 24.56 & 1.98 & -0.20 & 4.19 & 1.11 & $0.32 * *$ & 2.61 & 0.76 & 0.01 \\
\hline Lakshmi $\odot$ & 61.87 & 10.55 & 6.39 & 4.56 & 1.13 & $0.22 *$ & 29.89 & $1.89 *$ & -0.41 & 4.43 & 0.63 & -0.03 & 2.05 & 0.61 *** & -0.02 \\
\hline US-618 @) & 80.93 & 3.62 & $70.28^{*}$ & 4.54 & 2.02 & 0.09 & 31.22 & 0.15 & $18.62 * *$ & 4.23 & 0.77 & -0.01 & 2.26 & 1.26 & 0.03 \\
\hline Mean & 68.86 & 1.00 & & 4.58 & 1.00 & & 28.15 & 1.00 & & 4.01 & 1.00 & & 2.26 & 1.00 & \\
\hline S.Em \pm & 8.70 & 13.17 & & 0.46 & 0.84 & & 1.73 & 0.93 & & 0.23 & 0.32 & & 0.24 & 0.26 & \\
\hline
\end{tabular}


Table.3 Mean performance and stability parameters for yield and its components in tomato

\begin{tabular}{|c|c|c|c|c|c|c|c|c|c|c|c|c|}
\hline \multirow[t]{2}{*}{ Parents $(\mathrm{P}) /$ Crosses $(\mathrm{C})$} & \multicolumn{3}{|c|}{ Fruit set (\%) } & \multicolumn{3}{|c|}{ Days to first fruit harvest } & \multicolumn{3}{|c|}{ Days to last fruit harvest } & \multicolumn{3}{|c|}{ Fruit yield per plant (kg) } \\
\hline & $\mu$ Mean & $\mathbf{b}_{\mathrm{i}}$ & $\mathbf{S}^{2} \mathbf{d}_{\mathrm{i}}$ & $\mu$ Mean & $\mathbf{b}_{\mathbf{i}}$ & $\mathbf{S}^{2} \mathbf{d}_{\mathbf{i}}$ & $\mu$ Mean & $\mathbf{b}_{\mathbf{i}}$ & $\mathbf{S}^{2} \mathbf{d}_{\mathbf{i}}$ & $\mu$ Mean & $\mathbf{b}_{\mathbf{i}}$ & $\mathbf{S}^{2} \mathbf{d}_{\mathbf{i}}$ \\
\hline LE-53 (P1) & 48.20 & 0.92 & 3.92 & 69.00 & 1.24 & $29.83 * *$ & 143.80 & $0.89 *$ & -4.33 & 1.64 & 0.79 & 0.00 \\
\hline LE-62 (P2) & 51.84 & 1.01 & -4.50 & 68.11 & 1.13 & $10.96^{* *}$ & 139.70 & 1.27 & 5.87 & 2.14 & 1.22 & -0.01 \\
\hline LE-64 (P3) & 56.62 & 0.78 & -0.08 & 67.22 & 1.17 & $3.25^{*}$ & 139.10 & 1.09 & $118.16^{* * *}$ & 2.18 & 1.19 & $2.83 * *$ \\
\hline Arka Alok (P4) & 60.99 & 1.39 & $41.25^{* * *}$ & 69.33 & 1.40 & 0.76 & 131.70 & 0.73 & $181.51^{* * *}$ & 2.14 & 0.98 & $2.09 * *$ \\
\hline Arka Meghali (P5) & 54.38 & 1.10 & $26.15^{*}$ & 69.33 & 0.62 & $10.31 * *$ & 136.10 & 0.54 & $146.16^{* * *}$ & 2.45 & 1.30 & $2.08 * *$ \\
\hline Arka Vikas (P6) & 56.48 & 1.00 & $210.63^{* *}$ & 70.00 & 0.90 & $16.58 * *$ & 149.30 & 1.13 & $16.04 *$ & 2.61 & 1.37 & $1.69 * *$ \\
\hline LE-53 × LE-62 (C1) & 46.20 & 0.62 & $280.13^{* *}$ & 70.67 & 0.75 & 1.07 & 144.00 & 0.72 & $32.93 * *$ & 2.20 & 1.33 & $1.04 * *$ \\
\hline LE-53 x LE-64 (C2) & 50.09 & 0.57 & $42.27 * *$ & 66.78 & 0.97 & $22.17 * *$ & 135.90 & 0.92 & -0.82 & 2.02 & 0.43 & $1.28^{* *}$ \\
\hline LE-53 × A. Alok (C3) & 59.01 & 0.40 & $21.01 *$ & 70.62 & 0.90 & $15.61 * *$ & 139.90 & 0.69 & $391.15^{* *}$ & 1.86 & 0.38 & $1.08^{* *}$ \\
\hline LE-53 × A. Meghali $(C 4)$ & 52.01 & $0.38 *$ & -4.51 & 67.56 & 0.81 & $13.35^{* *}$ & 131.00 & 0.89 & $27.64 * *$ & 2.30 & 0.95 & $1.39 * *$ \\
\hline LE-53 × A. Vikas (C5) & 58.69 & $1.23^{*}$ & -4.55 & 67.62 & 1.24 & 2.06 & 132.70 & 0.65 & $394.72 * *$ & 2.15 & 0.72 & -0.01 \\
\hline LE-62 × LE-53 (C6) & 52.72 & 1.05 & 9.10 & 64.56 & 0.94 & $21.25^{* *}$ & 138.40 & 1.03 & $205.54 * *$ & 2.56 & 1.50 & 0.02 \\
\hline LE-62 × LE-64 (C7) & 60.05 & 1.11 & $105.44^{* *}$ & 63.67 & 1.18 & $5.67 *$ & 146.60 & 1.12 & $17.56^{*}$ & 2.49 & 1.22 & $0.33^{* * *}$ \\
\hline LE-62 × A. Alok $($ C8) & 63.55 & 0.71 & $108.77^{* * *}$ & 62.67 & 1.28 & 2.40 & 140.40 & 1.13 & $52.58^{* *}$ & 2.40 & 1.08 & $0.09^{* *}$ \\
\hline LE-62 $\times$ A. Meghali (C9) & 55.22 & 0.36 & $127.95 * *$ & 64.81 & 1.00 & $16.07 * *$ & 139.00 & 0.83 & $360.74 * *$ & 2.44 & 0.37 & 0.00 \\
\hline LE-62 × A. Vikas (C10) & 52.69 & 0.94 & $114.75^{* * *}$ & 65.33 & 1.27 & $6.07 * *$ & 134.20 & 1.17 & -4.20 & 2.45 & 0.99 & $0.18^{* *}$ \\
\hline LE-64 × LE-53 (C11) & 61.47 & $1.39 *$ & -4.65 & 65.33 & 0.97 & $22.74 * *$ & 145.80 & 1.02 & -0.53 & 2.53 & 0.41 & $0.05 *$ \\
\hline LE-64 × LE-62 (C12) & 51.51 & 1.27 & 11.28 & 66.89 & 0.60 & -0.89 & 137.00 & 1.11 & $71.06^{* *}$ & 1.99 & 1.18 & 0.01 \\
\hline LE-64 × A. Alok $($ C13) & 52.08 & 1.01 & $20.97 *$ & 65.11 & 0.71 & 2.05 & 137.00 & 0.95 & $63.96^{* *}$ & 2.29 & 0.81 & 0.03 \\
\hline LE-64 × A. Meghali (C14) & 53.70 & 1.32 & $31.15^{* *}$ & 63.67 & 1.14 & 1.99 & 138.40 & 1.16 & $81.56^{* *}$ & 2.56 & 0.87 & 0.03 \\
\hline LE-64 × A.Vikas (C15) & 54.28 & 1.15 & $36.62 * *$ & 64.89 & 1.40 & $20.85^{* *}$ & 140.80 & 0.99 & $106.83^{* *}$ & 2.02 & 0.98 & $0.07 *$ \\
\hline A. Alok $\times$ LE-53 (C16) & 57.42 & 0.76 & 11.24 & 68.33 & 0.99 & $53.05 * *$ & 142.70 & 0.84 & 2.79 & 2.32 & 1.08 & $1.02 * *$ \\
\hline A. Alok $\times$ LE-62 (C17) & 56.54 & 1.11 & 2.53 & 67.56 & 1.07 & 0.75 & 130.20 & 0.83 & 3.78 & 2.45 & 0.83 & $0.38^{* *}$ \\
\hline A. Alok $\times$ LE-64 $($ C18) & 50.75 & 1.00 & $29.53 * *$ & 68.44 & 0.70 & 2.72 & 138.10 & 0.88 & $56.71 * *$ & 2.23 & 1.46 & 0.57 ** \\
\hline A. Alok $\times$ A. Meghali $($ C19) & 54.25 & 1.20 & $18.80 *$ & 67.33 & 0.97 & $42.51 * *$ & 130.80 & 0.76 & $21.52 *$ & 2.15 & 0.95 & $0.56^{* *}$ \\
\hline A. Alok $\times$ A. Vikas $(\mathrm{C} 20)$ & 53.59 & 0.83 & 9.05 & 68.00 & 1.33 & 0.14 & 134.20 & 0.96 & 1.28 & 2.26 & 1.09 & $1.43^{* *}$ \\
\hline A. Meghali × LE-53 (21) & 58.08 & 0.89 & $38.01 * *$ & 66.96 & 0.82 & $12.94 * *$ & 138.40 & 1.39 & $116.86^{* *}$ & 2.74 & 0.84 & $2.02 * *$ \\
\hline A. Meghali $\times$ LE-62 (C22) & 48.20 & $1.33 *$ & -4.66 & 67.00 & 0.86 & -0.54 & 135.20 & 1.50 & 1.07 & 2.26 & 0.97 & $0.70^{* *}$ \\
\hline A. Meghali × LE-64 $(\mathrm{C} 23)$ & 59.99 & 1.18 & 12.45 & 64.67 & 1.22 & -0.69 & 143.90 & 1.36 & $16.59 *$ & 3.36 & 1.33 & 0.02 \\
\hline A. Meghali × A. Alok (C24) & 62.53 & 1.35 & 8.78 & 66.22 & 1.19 & 0.50 & 137.90 & 1.18 & $50.77 * *$ & 2.53 & 0.89 & -0.01 \\
\hline A. Meghali × A. Vikas $($ C25) & 59.09 & 0.92 & -4.40 & 68.30 & 0.64 & 2.43 & 142.80 & 1.24 & $147.65^{* *}$ & 2.93 & 0.81 & $0.67 * *$ \\
\hline A. Vikas $\times$ LE-53 (C26) & 58.70 & 1.44 & $57.89 * *$ & 66.78 & 0.53 & -0.57 & 149.60 & 1.06 & -3.86 & 2.92 & 1.33 & $0.15^{* *}$ \\
\hline A. Vikas × LE-62 (27) & 54.97 & 1.27 & -1.20 & 64.56 & 0.94 & $44.57 * *$ & 150.20 & 1.06 & -4.32 & 2.50 & 0.89 & $0.12^{* *}$ \\
\hline A. Vikas $\times$ LE-64 (C28) & 55.43 & 1.02 & $30.91 * *$ & 66.33 & 1.09 & 0.36 & 149.10 & 1.14 & 0.93 & 2.70 & 1.10 & $0.33^{* *}$ \\
\hline A. Vikas $\times$ A. Alok $($ C29) & 63.84 & 0.56 & $16.29^{*}$ & 67.06 & 1.22 & 1.12 & 148.20 & 0.92 & -2.30 & 3.06 & 1.30 & -0.01 \\
\hline A. Vikas $\times$ A. Meghali $($ C30) & 61.13 & 1.45 & $39.51 * *$ & 63.22 & 0.88 & $10.82 * *$ & 153.20 & 0.78 & $27.98 * *$ & 3.41 & 1.05 & 0.04 \\
\hline Lakshmi $\odot$ & 48.65 & 0.77 & $56.08 * *$ & 68.11 & 1.04 & $3.82^{*}$ & 150.90 & 1.05 & -4.23 & 2.23 & 1.06 & $0.09 * *$ \\
\hline US-618 @ & 55.36 & $1.24^{*}$ & -4.61 & 67.84 & 0.93 & $3.51 *$ & 150.00 & 1.06 & -4.31 & 2.30 & 0.97 & $0.15^{* *}$ \\
\hline Mean & 55.53 & 1.00 & & 66.838 & 1 & & 140.700 & 1 & & 2.414 & 1 & \\
\hline S.Em \pm & 4.68 & 0.28 & & 2.41 & 0.34 & & 6.100 & 0.5 & & 0.552 & 0.729 & \\
\hline
\end{tabular}

*,** Significant @ 5\% and 1\% level respectively. 
These results are in accordance with the observations made by Pandey et al., (2000), Joshi (2002), Hossain et al., (2006) and Alok and Singh (2009) for significant nonlinear G $\times \mathrm{E}$ interactions for days to $50 \%$ flowering.

\section{Number of flowers per cluster}

Among the stable parents, none could record significant higher no. of flowers than checks i.e. Lakshmi (4.43) and US-618 (4.23). The parents, LE-53 (4.13), LE-64 (4.02) and Arka Meghali (4.09) registered at par for no. of flowers per cluster over check US-618 with $\mathrm{S}^{2} \mathrm{~d}_{\mathrm{i}}$ near to zero and unit regression coefficient $\left(b_{i}\right)$ indicated the average stability for no. of flowers per cluster.

Among the hybrids, twenty four hybrids exhibited unit regression coefficient $\left(b_{i}\right)$ and near to zero deviation from regression $\left(\mathrm{S}^{2} \mathrm{~d}_{\mathrm{i}}\right)$ values considered to have wide adaptability to different environments with average stability. The hybrids, Arka Alok $\times$ LE-64 (4.56), LE$62 \times$ Arka Alok (4.35) and Arka Alok $\times$ Arka Vikas (4.24) recorded more and at par for no. of flowers per cluster than check US-618 (4.23) with $\mathrm{bi}=1$ and $\mathrm{S}^{2} \mathrm{~d}_{\mathrm{i}}=$ zero. Hence, they were considered to be stable hybrids whose performance does not change over different environments. One hybrid, Arka Alok $\times$ LE53 (4.10) expressed greater than unit regression coefficient (bi $>1)$ hence, specifically adapted to favorable environments. Similar results were reported by Alok and Singh (2009) in tomato.

\section{Number of fruits per cluster}

Among the parents, Arka Alok (2.26) recorded equal no. of fruits per cluster with best check US-618 and unit regression coefficient suggesting average stability for no. of fruits per cluster.

Among the eighteen stable $\left(\mathrm{S}^{2} \mathrm{~d}_{\mathrm{i}}=0\right)$ hybrids, Arka Vikas $\times$ Arka Meghali (2.61) recorded significant higher no. of fruits per cluster over best check US-618 (2.26) coupled with unit regression coefficient $\left(b_{i}\right)$ indicating superior and average stable hybrid which can be recommended for wider environments. The hybrids, LE-64 $\times$ Arka Meghali (2.42) followed by Arka Alok $\times$ LE-62 (2.38), LE$64 \times$ Arka Alok (2.30) recorded at par and more no. of fruits per plant than best check US-618 with average stability. The hybrids, Arka Alok $\times$ LE-64 (2.27) followed by LE-64 $\times$ LE-62 (2.19) and Arka Vikas $\times$ LE-53 (2.05) were at par with best check for no. of fruits per cluster and had greater than one regression value. Hence it can be recommended as stable hybrid for favourable environment. The hybrid LE-53×Arka Meghali (1.97) with regression coefficient significantly lower than unity indicating above average stability and specifically adapted to poor environments for this trait. These results are in accordance with the observations made by Alok and Singh (2009).

\section{Fruit set (\%)}

Among the parents, LE-64 (56.62 \%) recorded higher and at par fruit set over best check US-618 (55.36 \%) followed by LE-62 $(51.84 \%)$ and LE-53 (48.20 \%) with unit regression coefficient and $\mathrm{S}^{2} \mathrm{~d}_{\mathrm{i}}$ near to zero which can be adaptable to wider environments for this trait.

Among the hybrids, Arka Meghali $\times$ Arka Alok (62.53\%) recorded significant higher fruit set than best check US-618 (55.36\%) followed by Arka Meghali $\times$ LE-64 (59.99\%), Arka Meghali $\times$ Arka Vikas (59.09\%), Arka Alok $\times$ LE-53 (57.42\%), Arka Alok $\times$ LE-62 $(56.54 \%)$ recorded at par higher fruit set with check US-618 and unit regression coefficient were found to be with average stability and proposed for wider environments. LE-64 $\times$ LE-53 (61.47\%), LE-53 × Arka Vikas $(58.69 \%)$ and Arka Meghali $\times$ LE-62 $(48.20 \%)$ were found to be specifically 
adapted to favorable environments because of regression coefficient more than unity and with minimum deviation from regression for fruit set. The hybrid LE-53 $\times$ Arka Meghali $(52.01 \%)$ possess more than average stability (bi $>1$ and $\mathrm{S}^{2} \mathrm{~d}_{\mathrm{i}}=0$ ) and it can be adaptable to poor environments for this trait. This is in agreement with the findings of Arora et al., (1982) and Jyothi et al., (2012), Alok and Singh (2009) in tomato.

\section{Days to first fruit harvest}

Among the parents, Arka Alok (69.33 days) recorded less number of days to first fruit harvest and was at par with best check US618 (67.84 days) with unit regression value of one and was rated as widely adaptable with the average stability.

Among the stable hybrids, three hybrids viz., LE-62 $\times$ Arka Alok (62.67 days), LE-64 $\times$ Arka Meghali (63.67 days) and Arka Meghali $\times$ LE-64 (64.67 days) with significantly low mean value for days to first fruit harvest compared to best check US-618 (67.84 days) recorded unit regression coefficient $\left(b_{i}\right)$ and hence possess the average stability and widely adaptable to all environments. Similar results were reported by Cuartero and Cubero (1982) and Alok and Singh (2009) for days to first fruit harvest.

\section{Days to last fruit harvest}

Among the parents, LE-62 (139.70 days) recorded less no. of days to last fruit harvest over best check Lakshmi (150.90 days) with unit regression and it is rated with wide adaptability with the average stability.

While the parent LE-53 (143.80 days) recorded less than one regression coefficient $\left(b_{i}<1\right)$ and $S^{2} d_{i}=0$ thus possessed more than the average stability and adaptability to poor environments.
Among the stable genotypes, three hybrids viz., Arka Vikas $\times$ LE-62 (150.20 days), Arka Vikas $\times$ LE-64 (149.10 days) and Arka Vikas $\times$ LE-53 (149.60 days) recorded statistically at par for days to last fruit harvest with best check Lakshmi (150.90) and unit regression coefficient values $\left(b_{i}=1\right)$, hence can be adaptable to different environments with average stability. Similar results were reported by Joshi (2002) and Alok and Singh (2009) (Table 3).

\section{Fruit yield per plant (kg)}

Among the stable parents, LE-62 $(2.14 \mathrm{~kg})$ statistically at par for yield per plant with best check US-618 $(2.30 \mathrm{~kg})$ and unit regression coefficient $\left(b_{i}=1\right)$ indicating average stability and can be recommended for wider environments for yield per plant.

Among the stable hybrids, three hybrids viz., Arka Vikas $\times$ Arka Meghali (3.41 kg), Arka Meghali $\times$ LE-64 $(3.36 \mathrm{~kg})$ and Arka Vikas $\times$ Arka Alok (3.06 kg) have recorded significantly more yield per plant over the best check US-618 (2.30 kg) and regression coefficient value equal to unit $\left(b_{i}=1\right)$ hence, considered to be stable hybrids and can be recommended for wider environments. Similar results were also reported by Joshi (2002), Prasanna et al., (2007), Alok and Singh (2009), Ramesh et al., (2010) and Jyothi et al., (2012) for this trait.

Based on stability analysis, the hybrids viz., Arka Vikas $\times$ Arka Meghali, Arka Meghali $\times$ LE-64, Arka Vikas $\times$ Arka Alok, LE-62 $\times$ LE-53, LE-64 $\times$ Arka Meghali, Arka Meghali $\times$ Arka Alok and LE-62 $\times$ Arka Meghali were identified as stable and best performing hybrids for fruit yield per plant and other traits hence, suitable for wider environments.

Among the parents, LE-62 was the stable with high per se performance for yield per plant. 
References

Alok, T., and Singh, J. P. 2009. Stability analysis for growth, yield and quality characters of tomato (Solanum lycopersicum L). Pantnagar J. Res., 7(2): 180-183.

Arora, S.K., Pandit, M.L. and Singh, K. 1982. Evaluation of tomato varieties under hot weather conditions. Haryana J. Hort. Sci. 11: 101-111.

Cuartero, J., and Cubero, J.L. 1982. Genotype-environment interaction in tomato. Theor. App. Genet. 61: 273277.

Eberhart, S.A., and Russell, W.A. 1966. Stability parameters for comparing varieties. Crop Science 6: 36 -40.

FAOSTAT, 2015. Statistical Databases. Food and Agriculture Organization of the United Nations, Statistics Division, Rome, Italy.

Hossain, H., Rahman, M.A and Hossain, M.A. 2006. Genotype $\times$ Environment response and stability assessment in tomato (Lycopersicon esculentum Mill.). SAARC J. of agri. 4, 13-19.

Joshi, A., 2002. Stability analysis for yield and quality characters in tomato (Lycopersicon esculentum Mill.). Ph.D. thesis, GPBAU, Pantnagar.

Jyothi, H.K., Patil, M.G. and Santhosha, H.M. 2012. Studies on stability of processingtype genotypes of tomato (Solanum lycopersicum L.). J. Hortl. Sci. 7(2): $138-141$.

Kalloo, G., Chaurasia, S.N.S and Singh, M. 1998. Stability analysis in tomato. Veg. Sci. 25: 81-84.

Mandal, A.R., Senapati, B.K and Maity, T.K.
2000.

Genotype-environment interactions, stability and adaptability of tomato (Lycopersicon esculentum Mill.). Veg. Sci. 27:155-157.

Mulge, R., and Aravindakumar, J.S. 2003. Stability analysis for growth and earliness in tomato. Indian J. Hort. 60: 353-356.

NHB, 2015. Indian Horticulture Database, NHB, Gurgaon, Haryana.Ortiz, R. and Izquierdo, J., 1994, Yield stability differences among tomato genotypes grown in Latin America and the Caribbean. HortScience, 29: 1175-1177.

Pandey, S., Dixit, J, Dwivedi, S. V and Dubey, R. 2000. Stability analysis for yield and its components in tomato (Lycopersicon esculentum Mill.). Haryana J. Hortic. Sci. 29(3/4): 207208.

Pandey, S.C., 1983. Stability analysis in tomato (Lycopersicon esculentum Mill.). Indian J. Agric. Res. 17: 229233.

Panse, V.G., and Sukhatme, P.V. (Revised by Sukhatme, P.V. and Amble, V.N.) 1985. Statistical Methods for Agricultural Workers. ICAR, New Delhi. 187-202.

Prasanna, H.C., Chaubey, T, Kumar, R, Rai, M, Verma, A and Singh, S. 2007. Identification of stable variety for yield and quality attributes in tomato. Veg. Sci. 34: 131-134.

Ramesh, M., Sridevi, O, Salimath, P.M, Deshpande, S.K, Khot, A.B. 2010. Performance and stability of different tomato (Solanum lycopersicum) genotypes. Indian J. of Agric. Sci. 80(10): 898-901.

\section{How to cite this article:}

Shankar, A., R.V.S.K. Reddy, P. Saidaiah, K. Uma Krishna and Uma Jyothi, K. 2017. Study on Stability for Yield and Its Components in Tomato (Solanum lycopersicum L.). Int.J.Curr.Microbiol.App.Sci. 6(9): 804-812. doi: https://doi.org/10.20546/ijcmas.2017.609.099 